\title{
EM-Based Optimization Exploiting Partial Space Mapping and Exact Sensitivities
}

\author{
John W. Bandler, Ahmed S. Mohamed, Mohamed H. Bakr, Kaj Madsen and Jacob Søndergaard \\ McMaster University, Hamilton, ON, Canada L8S 4K1, www.sos.mcmaster.ca
}

\begin{abstract}
We present a family of robust techniques for exploiting sensitivities in EM-based circuit optimization through Space Mapping (SM). We utilize derivative information for parameter extractions and mapping updates. We exploit a Partial Space Mapping (PSM) concept where a reduced set of parameters is sufficient for parameter extraction optimization. Upfront gradients of both EM (fine) model and coarse surrogates can initialize possible mapping approximations. Illustrations include a two-section 10:1 impedance transformer and a microstrip bandstop filter.
\end{abstract}

\section{INTRODUCTION}

The SM approach [1] involves a suitable calibration of a fine model by a physically-based "coarse" surrogate. The fine model may be time intensive and field theoretic and accurate, while the surrogate is a faster (less accurate) representation.

We present, for the first time, new techniques for exploiting exact sensitivities in EM-based circuit design in the context of SM technology. If the EM simulator is capable of providing gradient information, these gradients can be exploited to enhance a coarse surrogate. New approaches for utilizing derivatives in the parameter extraction process and mapping update are presented.

An efficient procedure exploiting a PSM concept [2] is proposed. Several approaches for utilizing sensitivities and PSM are suggested.

Alessandri et al. spurred the recent application of the adjoint network method using a mode matching orientation [3]. Currently, we are developing the adjoint technique within a method of moments environment [4]. These techniques facilitate powerful gradient-based optimizers. Our new work complements these efforts at gradient estimation for design optimization using EM simulations.

This work was supported in part by the Natural Sciences and Engineering Research Council of Canada under Grants OGP0007239 and STR234854-00, through the Micronet Network of Centres of Excellence and Bandler Corporation.

J.W. Bandler is also with Bandler Corporation, P.O. Box 8083, Dundas, Ontario, Canada L9H 5E7.

K. Madsen and J. Søndergaard are with the Department of Mathematical Modelling, Technical University of Denmark, DK-2800 Lyngby, Denmark.

\section{Aggressive Space MAPPING}

\section{A. Original Design Problem}

The original design problem is

$$
\boldsymbol{x}_{f}^{*}=\arg \min _{\boldsymbol{x}_{f}} U\left(\boldsymbol{r}_{f}\left(\boldsymbol{x}_{f}\right)\right)
$$

Here, the fine model response vector is denoted by $\boldsymbol{r}_{f}$ $\in \mathfrak{R}^{m \times 1}$, e.g., $\left|S_{11}\right|$ at selected frequency points; $m$ is the number of sample points; the fine model point is denoted $\boldsymbol{x}_{f} \in \mathfrak{R}^{n \times 1}$, where $n$ is the number of design parameters. $U$ is a suitable objective function. $\boldsymbol{x}_{f}^{*}$ is the optimal design.

\section{B. Parameter Extraction (PE)}

PE is crucial to SM: we extract a coarse model corresponding to a fine model response. For PE we designate a complete set of basic responses by $\boldsymbol{R} \in \mathfrak{R}^{M \times 1}$, not necessarily identical to $\boldsymbol{r}$, where $M$ is the product of number of simulation frequency points and number of basic responses. Fine and coarse response vectors are denoted by $\boldsymbol{R}_{f}$ and $\boldsymbol{R}_{c}$, respectively. For example, we can use real and imaginary parts of $S$ parameters.

\section{Aggressive Space Mapping Approach}

Aggressive SM solves the nonlinear system

$$
\begin{aligned}
\boldsymbol{f} & =\boldsymbol{P}\left(\boldsymbol{x}_{f}\right)-\boldsymbol{x}_{c}^{*}=\mathbf{0} \\
& =\boldsymbol{x}_{c}-\boldsymbol{x}_{c}^{*}=\mathbf{0}
\end{aligned}
$$

for $\boldsymbol{x}_{f}$, where $\boldsymbol{P}$ is a mapping between the two model spaces and $\boldsymbol{x}_{c} \in \mathfrak{R}^{n \times 1}$. First-order Taylor approximations are given by

$$
\begin{aligned}
& \boldsymbol{P}\left(\boldsymbol{x}_{f}\right) \approx \boldsymbol{P}\left(\boldsymbol{x}_{f}^{(j)}\right)+\boldsymbol{J}_{P}\left(\boldsymbol{x}_{f}^{(j)}\right)\left(\boldsymbol{x}_{f}-\boldsymbol{x}_{f}^{(j)}\right) \\
& \boldsymbol{x}_{c} \approx \boldsymbol{x}_{c}^{(j)}+\left.\boldsymbol{J}_{P}\left(\boldsymbol{x}_{f}^{(j)}\right)\left(\boldsymbol{x}_{f}-\boldsymbol{x}_{f}^{(j)}\right)\right|_{\text {Through PE }}
\end{aligned}
$$

where the Jacobian of $\boldsymbol{P}$ at the $j$ th iteration is expressed by

$$
\boldsymbol{J}_{P}^{(j)} \triangleq \boldsymbol{J}_{P}\left(\boldsymbol{x}_{f}^{(j)}\right)=\left(\frac{\partial \boldsymbol{P}^{T}}{\partial \boldsymbol{x}_{f}}\right)_{\boldsymbol{x}_{f}=\boldsymbol{x}_{f}^{(j)}}^{T}=\left.\left(\frac{\partial \boldsymbol{x}_{c}^{(j) T}}{\partial \boldsymbol{x}_{f}}\right)\right|_{P E}
$$


We designate an approximation to this Jacobian by the square matrix $\boldsymbol{B} \in \mathfrak{R}^{n \times n}$, i.e., $\boldsymbol{B} \approx \boldsymbol{J}_{p}\left(\boldsymbol{x}_{f}\right)$.

From (2) and (3b) we can formulate the system

$$
\begin{aligned}
& \left(\boldsymbol{x}_{c}^{(j)}-\boldsymbol{x}_{c}^{*}\right)+\boldsymbol{B}^{(j)}\left(\boldsymbol{x}_{f}^{(j+1)}-\boldsymbol{x}_{f}^{(j)}\right)=\mathbf{0} \\
& \boldsymbol{f}^{(j)}+\boldsymbol{B}^{(j)} \boldsymbol{h}^{(j)}=\mathbf{0}
\end{aligned}
$$

Solving (5b) for $\boldsymbol{h}^{(j)}$, the quasi-Newton step provides the next tentative iterate $\boldsymbol{x}_{f}^{(j+1)}$

$$
\boldsymbol{x}_{f}^{(j+1)}=\boldsymbol{x}_{f}^{(j)}+\boldsymbol{h}^{(j)}
$$

\section{A PRoposed Algorithm}

\section{A. PE Exploiting Sensitivity}

Through the traditional PE process we can obtain the point $\boldsymbol{x}_{c}$ that corresponds to $\boldsymbol{x}_{f}$ such that

$$
\boldsymbol{R}_{f} \approx \boldsymbol{R}_{c}
$$

Differentiating both sides of (7) w.r.t. $\boldsymbol{x}_{f}$, we obtain

$$
\left(\frac{\partial \boldsymbol{R}_{f}^{T}}{\partial \boldsymbol{x}_{f}}\right)^{T} \approx\left(\frac{\partial \boldsymbol{R}_{c}^{T}}{\partial \boldsymbol{x}_{c}}\right)^{T}\left(\frac{\partial \boldsymbol{x}_{c}^{T}}{\partial \boldsymbol{x}_{f}}\right)^{T}
$$

Using (4) the relation (8) can be simplified to

$$
\boldsymbol{J}_{f} \approx \boldsymbol{J}_{c} \boldsymbol{B}
$$

where $\boldsymbol{J}_{f}$ and $\boldsymbol{J}_{c}$ are the fine and coarse Jacobians at $\boldsymbol{x}_{f}$ and $\boldsymbol{x}_{c}$, respectively $\left(\boldsymbol{J}_{f}, \boldsymbol{J}_{c} \in \mathfrak{R}^{M \times n} ; M \geq n\right)$. Solving (9) for $\boldsymbol{B}$ yields a least squares solution.

At the $j$ th iteration we obtain $\boldsymbol{x}_{c}{ }^{(j)}$ through a Gradient Parameter Extraction (GPE) process:

$$
\boldsymbol{x}_{c}^{(j)}=\arg \min _{\boldsymbol{x}_{c}}\left\|\left[\begin{array}{llll}
\boldsymbol{e}_{0}^{T} & \lambda \boldsymbol{e}_{1}^{T} & \cdots & \lambda \boldsymbol{e}_{n}^{T}
\end{array}\right]^{T}\right\|, \lambda \geq 0
$$

where $\lambda$ is a weighting factor and $\boldsymbol{E}=\left[\begin{array}{lll}\boldsymbol{e}_{1} & \boldsymbol{e}_{2} \ldots \boldsymbol{e}_{n}\end{array}\right]$.

$$
\begin{aligned}
& \boldsymbol{e}_{0}=\boldsymbol{R}_{f}\left(\boldsymbol{x}_{f}^{(j)}\right)-\boldsymbol{R}_{c}\left(\boldsymbol{x}_{c}\right) \\
& \boldsymbol{E}=\boldsymbol{J}_{f}\left(\boldsymbol{x}_{f}^{(j)}\right)-\boldsymbol{J}_{c}\left(\boldsymbol{x}_{c}\right) \boldsymbol{B}
\end{aligned}
$$

\section{B. Partial Space Mapping (PSM)}

Consider utilizing a subset of the physical parameters in the coarse space $\boldsymbol{x}_{c}^{P S M} \in \mathfrak{R}^{k \times 1}, k \leq n$. PSM is illustrated in Fig. 1. It can be represented by

$$
\boldsymbol{x}_{c}=\left[\begin{array}{c}
\boldsymbol{x}_{c}^{P S M} \\
\boldsymbol{x}_{f}^{s}
\end{array}\right]=\left[\begin{array}{c}
\boldsymbol{P}_{P S M}\left(\boldsymbol{x}_{f}\right) \\
\boldsymbol{x}_{f}^{S}
\end{array}\right]
$$

In this context (9) becomes

$$
\boldsymbol{J}_{f} \approx \boldsymbol{J}_{c}^{P S M} \boldsymbol{B}^{P S M}
$$

where $\boldsymbol{B}^{P S M} \in \mathfrak{R}^{k \times n}$ and $\boldsymbol{J}_{c}^{P S M} \in \mathfrak{R}^{M \times k}$ is the Jacobian of the coarse model at $\boldsymbol{x}_{c}^{P S M}$. Solving (13) for $\boldsymbol{B}^{P S M}$ yields the least squares solution at the $j$ th iteration

$$
\boldsymbol{B}^{P S M(j)}=\left(\boldsymbol{J}_{c}^{P S M(j) T} \boldsymbol{J}_{c}{ }^{P S M(j)}\right)^{-1} \boldsymbol{J}_{c}^{P S M(j) T} \boldsymbol{J}_{f}^{(j)}
$$

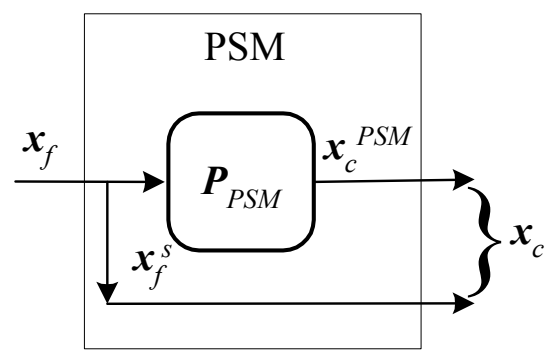

Fig. 1 Partial Space Mapping (PSM).

Relation ( $5 \mathrm{~b}$ ) becomes underdetermined. The minimum norm solution for $\boldsymbol{h}^{(j)}$ is given by

$$
\boldsymbol{h}_{\text {min norm }}^{(j)}=\boldsymbol{B}^{P S M(j) T}\left(\boldsymbol{B}^{P S M(j)} \boldsymbol{B}^{P S M(j) T}\right)^{-1}\left(-\boldsymbol{f}^{(j)}\right)
$$

\section{Mapping Update Alternatives}

If we have exact derivatives throughout, we can use them to obtain $\boldsymbol{B}$ at each iteration in the PE. Note that this matrix can be iteratively fed back into the GPE process and refined before making a step in the fine model space. We can also use (14) to update $\boldsymbol{B}^{P S M(j)}$.

If we do not have exact derivatives, various approaches to initializing or constraining $\boldsymbol{B}$ and $\boldsymbol{B}^{P S M(1)}$ can be devised, for example, we can use finite differences. Either matrix may be updated using a Broyden update. Hybrid schemes can be formally developed following the integrated gradient approximation approach to optimization by Bandler et al. [5].

On the assumption that the fine and coarse models share the same physical background, Bakr et al. [6] suggested that $\boldsymbol{B}$ could be better conditioned, in the PE process, if it is constrained to be close to the identity matrix $I$ by

$$
\boldsymbol{B}=\arg \min _{\boldsymbol{B}}\left\|\left[\boldsymbol{e}_{1}^{T} \cdots \boldsymbol{e}_{n}^{T} \eta \Delta \boldsymbol{b}_{1}^{T} \cdots \eta \Delta \boldsymbol{b}_{n}^{T}\right]^{T}\right\|_{2}^{2}
$$

where $\eta$ is a weighting factor, $\boldsymbol{e}_{i}$ and $\Delta \boldsymbol{b}_{i}$ are the $i$ th columns of $\boldsymbol{E}$ and $\Delta \boldsymbol{B}$, respectively, defined as

$$
\begin{aligned}
\boldsymbol{E} & =\boldsymbol{J}_{f}-\boldsymbol{J}_{c} \boldsymbol{B} \\
\Delta \boldsymbol{B} & =\boldsymbol{B}-\boldsymbol{I}
\end{aligned}
$$

The analytical solution of (16) is given by 


$$
\boldsymbol{B}=\left(\boldsymbol{J}_{c}^{T} \boldsymbol{J}_{c}+\eta^{2} \boldsymbol{I}\right)^{-1}\left(\boldsymbol{J}_{c}^{T} \boldsymbol{J}_{f}+\eta^{2} \boldsymbol{I}\right)
$$

\section{Proposed Algorithm}

Step 1 Set $j=1$. Initialize $\boldsymbol{B}=\boldsymbol{I}$ for the PE process. Obtain the optimal coarse model design $\boldsymbol{x}_{c}{ }^{*}$ and use it as the initial fine model point

$$
\boldsymbol{x}_{f}^{(1)}=\boldsymbol{x}_{c}^{*}=\underset{\boldsymbol{x}_{c}}{\arg \min } U\left(\boldsymbol{r}_{c}\left(\boldsymbol{x}_{c}\right)\right)
$$

Step 2 If derivatives exist execute GPE as in (10). Otherwise, execute the traditional PE where $\lambda=0$.

Step 3 Initialize the mapping matrix $\boldsymbol{B}^{P S M}$ using (14).

Step 4 Stop if

$$
\left\|\boldsymbol{f}^{(j)}\right\|<\varepsilon_{1} \text { or }\left\|\boldsymbol{R}_{f}^{(j)}-\boldsymbol{R}_{c}^{*}\right\|<\varepsilon_{2}
$$

Step 5 Evaluate $\boldsymbol{h}^{(j)}$ using (15).

Step 6 Find the next $\boldsymbol{x}_{f}^{(j+1)}$ using (6).

Step 7 Perform GPE or PE as in Step 2.

Step 8 If derivatives exist use (14) to obtain $\boldsymbol{B}^{P S M(j)}$. Otherwise update $\boldsymbol{B}^{P S M(j)}$ using a Broyden formula.

Step 9 Set $j=j+1$ and go to Step 4 .

The output of the algorithm is the fine space mapped optimal design $\overline{\boldsymbol{x}}_{f}$ and the mapping matrix $\boldsymbol{B}^{P S M}$.

\section{EXAMPLES}

\section{A. Capacitively Loaded 10:1 Impedance Transformer [7]}

We consider a "coarse" model as an ideal two-section transmission line (TL), where the "fine" model is a capacitively loaded TL with capacitors $C_{1}=C_{2}=C_{3}=10$ pF. Design parameters are normalized lengths $L_{1}$ and $L_{2}$, w.r.t. the quarter-wave length $L_{q}$ at the center frequency 1 $\mathrm{GHz}$, and characteristic impedances $Z_{1}$ and $Z_{2}$. Thus, $\boldsymbol{x}_{f}=$ $\left[\begin{array}{lll}L_{1} & L_{2} & Z_{1} Z_{2}\end{array}\right]^{T}$. Design specifications are

$$
\left|S_{11}\right| \leq 0.5, \text { for } 0.5 \mathrm{GHz} \leq \omega \leq 1.5 \mathrm{GHz}
$$

with eleven points per frequency sweep. We utilize the real and imaginary parts of $S_{11}$ in the GPE (10). We solve (10) using the Levenberg-Marquardt algorithm available in the Matlab ${ }^{\mathrm{TM}}$ Optimization Toolbox [8].

Case 1. We consider $\boldsymbol{x}_{c}^{P S M}=\left[\begin{array}{ll}L_{1} & L_{2}\end{array}\right]^{T}$ while $\boldsymbol{x}_{f}^{s}=\left[Z_{1}\right.$ $\left.Z_{2}\right]^{T}$ are kept fixed. We employed adjoint analysis [9] to obtain all Jacobians. We initialize $\boldsymbol{B}^{P S M}$ with (14). The algorithm converges in a single iteration ( 2 fine model evaluations). See Fig. 2. The final mapping is

$$
\boldsymbol{B}^{P S M}=\left[\begin{array}{cccc}
1.044 & -0.017 & 0.009 & 0.002 \\
-0.011 & 1.079 & -0.011 & 0.006
\end{array}\right]
$$

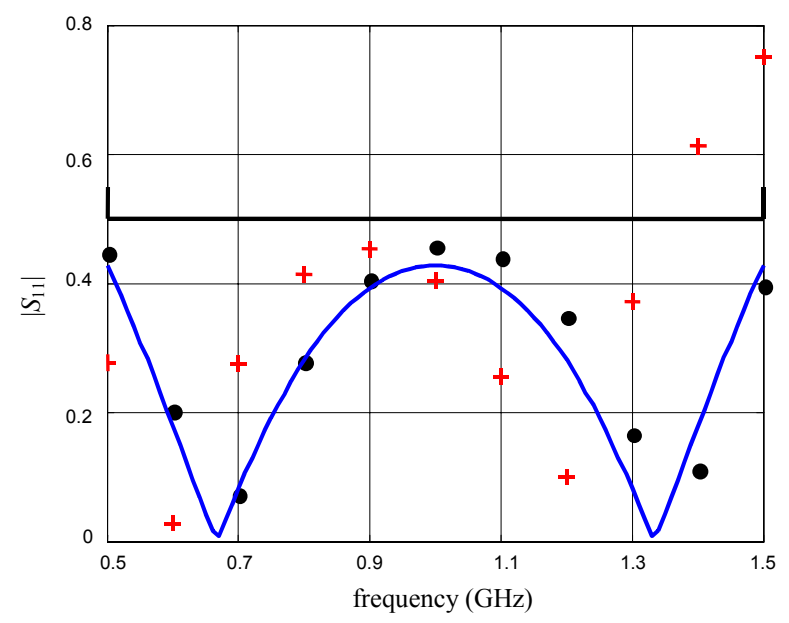

Fig. 2. Optimal coarse model target response (-), the fine model response at the starting point $(+)$ and final design $(\bullet)$ for the capacitively loaded 10:1 transformer with $L_{1}$ and $L_{2}$ as the PSM coarse model parameters.

Case 2. We also apply the algorithm for $\boldsymbol{x}_{c}^{P S M}=\left[L_{2}\right]$. The result is very similar to Fig. 2 . We also converge in a single iteration ( 2 fine model evaluations). The final mapping is

$$
\boldsymbol{B}^{P S M}=\left[\begin{array}{llll}
1.067 & 1.186 & -0.0027 & 0.0092
\end{array}\right]
$$

Case 3. We apply the algorithm for $\boldsymbol{x}_{c}^{P S M}=\left[L_{1}\right]$. The result is again similar to Fig. 2. Convergence is in a single iteration ( 2 fine model evaluations). The final mapping is

$$
\boldsymbol{B}^{P S M}=\left[\begin{array}{llll}
1.133 & 0.685 & 0.0092 & 0.00297
\end{array}\right]
$$

\section{B. Bandstop Microstrip Filter with Open Stubs [2]}

Our approach is applied to a symmetrical bandstop microstrip filter with three open stubs. The open stub lengths are $L_{1}, L_{2}, L_{1}$ and $W_{1}, W_{2}, W_{1}$ are the corresponding stub widths. An alumina substrate with thickness $H=25$ mil, width $W_{0}=25$ mil and dielectric constant $\varepsilon_{r}=9.4$ is used for a $50 \Omega$ feeding line. The design parameters are $\boldsymbol{x}_{f}=\left[\begin{array}{lllll}W_{1} & W_{2} & L_{0} & L_{1} & L_{2}\end{array}\right]^{T}$. The design specifications are

$$
\begin{array}{ll}
\left|S_{21}\right| \leq 0.05 & \text { for } 9.3 \mathrm{GHz} \leq \omega \leq 10.7 \mathrm{GHz} \text { and } \\
\left|S_{21}\right| \geq 0.9 & \text { for } 12 \mathrm{GHz} \leq \omega \text { and } \omega \leq 8 \mathrm{GHz}
\end{array}
$$

Sonnet's $\boldsymbol{e m}^{\mathrm{TM}}$ [10] driven by Empipe ${ }^{\mathrm{TM}}$ [11] is employed as the fine model, using a high-resolution grid with a 1 mil $\times 1$ mil cell size. As a coarse model we use simple transmission lines and classical formulas to calculate the characteristic impedance and the effective dielectric constant of each transmission line. We use OSA90/hope ${ }^{\mathrm{TM}}$ [11] built-in transmission line elements. 
Using OSA90/hope ${ }^{\mathrm{TM}} \boldsymbol{x}_{c}{ }^{*}=\left[\begin{array}{l}4.5609 .351 \\ 107.80\end{array} 111.03\right.$ $108.75]^{T}$ (in mils). We use 21 points per frequency sweep. We utilize the real and imaginary parts of $S_{11}$ and $S_{21}$ in the traditional PE, for which $\lambda=0$ in (10).

During the PE we consider $\boldsymbol{x}_{c}^{P S M}=\left[\begin{array}{ll}L_{1} & L_{2}\end{array}\right]^{T}$ while $\boldsymbol{x}_{f}^{S}=$ $\left[\begin{array}{lll}W_{1} & W_{2} & L_{0}\end{array}\right]^{T}$ are held fixed. Finite differences estimate the fine and coarse Jacobians. We initialize $\boldsymbol{B}$ with (14).

The algorithm converges in 5 iterations (6 fine model evaluations). See Fig. 3. Results are shown in Table I. The final mapping is

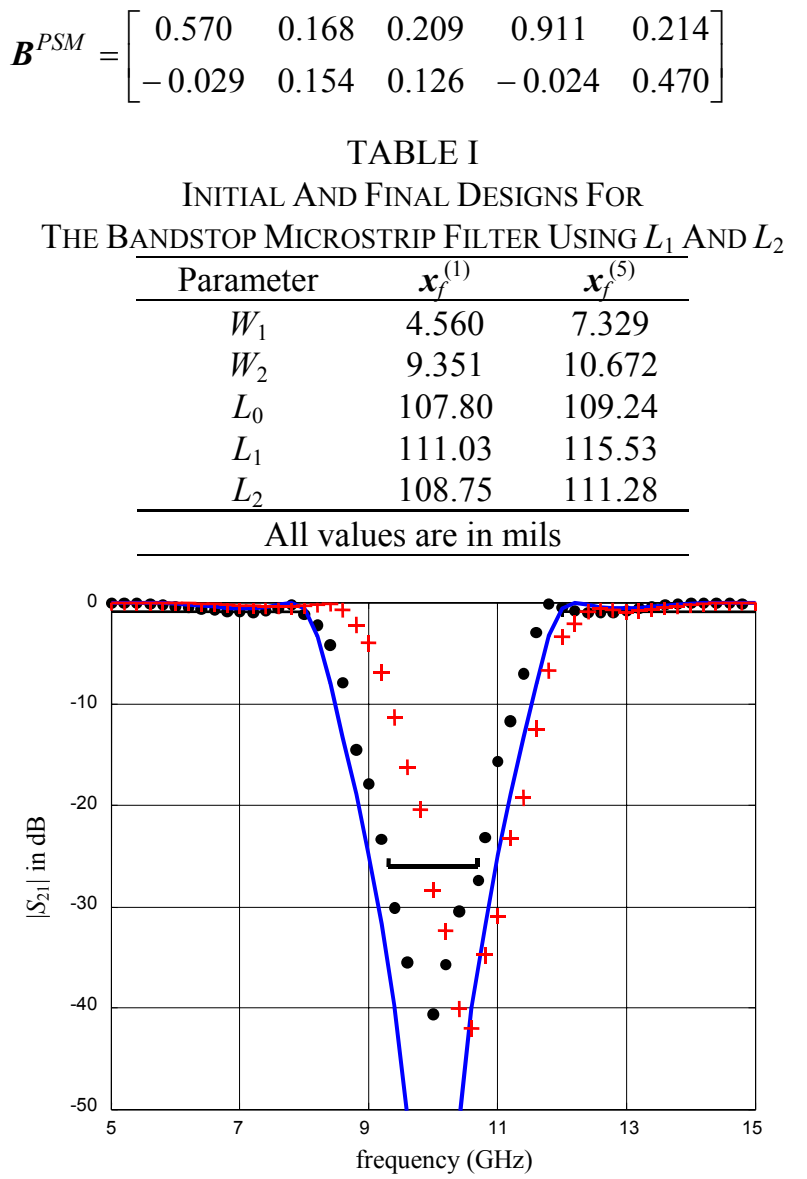

Fig. 3. Optimal OSA90/hope coarse response (-) and em fine model response at the starting point $(+)$ and at the final design $(\bullet)$ for the bandstop filter using a fine frequency sweep with $L_{1}$ and $L_{2}$ as the PSM coarse model parameters.

\section{CONCLUSIONS}

We present a family of robust techniques for exploiting sensitivities in EM-based circuit optimization through SM. We exploit a PSM concept where a reduced set of parameters is sufficient in the PE process. Available gradients can initialize mapping approximations. Exact or approximate Jacobians of responses can be utilized. For flexibility, we propose different possible "mapping matrices" for the PE processes and SM iterations. Broyden updates can be used for approximated Jacobians. Trust region methodologies can be employed. Our approaches have been tested on several examples.

Final mappings are useful in statistical analysis and yield optimization. Furthermore, the notion of exploiting reduced sets of physical parameters reflects the idea of postproduction tuning.

\section{ACKNOWLEDGEMENT}

The authors thank Dr. J.C. Rautio, President, Sonnet Software, Inc., Liverpool, NY, for making $\boldsymbol{e m}^{\mathrm{TM}}$ available.

\section{REFERENCES}

[1] J.W. Bandler, R.M. Biernacki, S.H. Chen, R.H. Hemmers and K. Madsen, "Electromagnetic optimization exploiting aggressive space mapping," IEEE Trans. Microwave Theory Tech., vol. 43, 1995, pp. 2874-2882.

[2] M.H. Bakr, J.W. Bandler, M.A. Ismail, J.E. Rayas-Sánchez and Q.J. Zhang, "Neural space-mapping optimization for EM-based design," IEEE Trans. Microwave Theory Tech., vol. 48, 2000, pp. 2307-2315.

[3] F. Alessandri, M. Mongiardo and R. Sorrentino, "New efficient full wave optimization of microwave circuits by the adjoint network method," IEEE Microwave and Guided Wave Letts., vol. 3, 1993, pp. 414-416.

[4] N. Georgieva, S. Glavic, M. Bakr and J.W. Bandler, "Feasible adjoint sensitivity technique for EM design optimization," IEEE IMS, Seattle, WA, 2002.

[5] J.W. Bandler, S.H. Chen, S. Daijavad and K. Madsen, "Efficient optimization with integrated gradient approximations," IEEE Trans. Microwave Theory Tech., vol. 36,1988 , pp. 444-455.

[6] M.H. Bakr, J.W. Bandler, K. Madsen and J. Søndergaard, "Review of the space mapping approach to engineering optimization and modeling," Optimization and Engineering, vol. 1, 2000, pp. 241-276.

[7] M.H. Bakr, J.W. Bandler, K. Madsen, J.E. Rayas-Sánchez and J. Søndergaard, "Space mapping optimization of microwave circuits exploiting surrogate models," IEEE Trans. Microwave Theory Tech., vol. 48, 2000, pp. $2297-$ 2306.

[8] Matlab ${ }^{\mathrm{TM}}$, Version 6.0, The MathWorks, Inc., 3 Apple Hill Drive, Natick MA 01760-2098, 2000.

[9] J.W. Bandler, "Computer-aided circuit optimization," in Modern Filter Theory and Design, G.C. Temes and S.K. Mitra, Eds. New York: Wiley, 1973, pp. 211-271.

[10] $\boldsymbol{e m}^{\mathrm{TM}}$ Version 5.1a, Sonnet Software, Inc., 1020 Seventh North Street, Suite 210, Liverpool, NY 13088, 1997.

[11] OSA90/hope ${ }^{\mathrm{TM}}$ and Empipe ${ }^{\mathrm{TM}}$ Version 4.0, formerly Optimization Systems Associates Inc., P.O. Box 8083, Dundas, Ontario, Canada L9H 5E7, 1997, now Agilent EEsof EDA, 1400 Fountaingrove Parkway, Santa Rosa, CA 95403-1799. 